Article

\title{
The Anti-Aging Potential of Extracts from Chaenomeles sinensis
}

\author{
Shintaro Itoh, Manami Yamaguchi, Keita Shigeyama and Ikuyo Sakaguchi *
}

Research and Development Department, Club Cosmetics Co., Ltd., Nara 630-0222, Japan;

sito@clubcosmetics.co.jp (S.I.); mayamagu@clubcosmetics.co.jp (M.Y.); kshigeya@clubcosmetics.co.jp (K.S.)

* Correspondence: ikuyos@clubcosmetics.co.jp; Tel.: +81-0743-76-2990

Received: 21 February 2019; Accepted: 14 March 2019; Published: 18 March 2019

\begin{abstract}
The Chaenomeles sinensis fruit is used as an effective antitussive agent, analgesic, and diuretic in traditional Chinese medicine. It has been reported that $C$. sinensis fruit extracts have antimicrobial and anti-inflammatory effects. However, there are very few reports about the effects of $C$. sinensis extracts on skin. In this study, we investigated the effect of $C$. sinensis extracts on skin aging. The results of in vitro assays showed that whole fruit extracts of $C$. sinensis had superoxide dismutase (SOD)-like activity and inhibited the activity of dermal extracellular matrix proteases: Elastase and collagenase. The inhibitory effect of the whole fruit (containing seeds) extract on elastase activity was higher than that of the sarcocarp (seeds removed) extract. Further, the sarcocarp extract showed a higher level of SOD-like activity and a greater inhibitory effect on collagenase activity than the whole fruit extract. In particular, among the three activities studied, the sarcocarp extract showed the most significant inhibitory effect on collagenase activity at low concentrations. The polyphenol-rich fraction obtained from the sarcocarp showed significant collagenase inhibition. Based on these results, we concluded that phenolic compounds from $C$. sinensis sarcocarp have the potential to protect against skin aging through anti-collagenase activity.
\end{abstract}

Keywords: collagenase inhibition; polyphenol; Chaenomels sinensis

\section{Introduction}

The skin is the largest organ of the human body and plays a major role in protecting the internal organs from the external environment. Skin aging is caused by both intrinsic damage due to chronological aging, and extrinsic damage such as dryness, sunlight, smoking, and environmental pollution. With respect to extrinsic damage, the effect of ultraviolet (UV) irradiation is well known and generally referred to as photo-aging [1]. UV irradiation leads to DNA damage and induces oxidative stress by the production of reactive oxygen species (ROS) [2,3]. These pathways increase the synthesis and activity of proteases that degrade the extracellular matrix (ECM), which comprises collagen and elastin fibers [4]. Takeuchi et al. reported that neutrophil elastase activity was increased in mouse skin following UV irradiation [5]. Fisher et al. reported that collagenase and 92-kDa gelatinase activities were increased in white adult skin after repeated exposure to UV corresponding to varied wavelengths (from 290-800 nm) [6]. In recent years, it has been reported that the effects of skin aging such as darkness, spots, and wrinkles are accelerated even with mild UV irradiation experienced in daily life rather than excessive sunburn, in long-term skin aging studies [7]. In addition to sunscreen agents, which protect skin from UV-irradiation, by absorbing or reflecting UV light, considerable attention is being paid to supplements that suppression of the formation of ROS and the subsequent activation of ECM-degrading proteases.

C. sinensis is a plant species of the family Rosaceae and is native to China. C. sinensis is widely cultivated in Japan, in the Kagawa, Ehime, Yamagata, Nagano, and Nara prefectures [8]. The fruit are 
not consumed fresh because they are hard and highly acidic. However, it is known that the C. sinensis fruit have a healing effect on cough and are used in the preparation of fruit liquor, jam, and candy. Dried C. sinensis fruit are marketed for their use in traditional Chinese medicine. C. sinensis fruit are rich in triterpenes and phenolic compounds including catechins and procyanidins, which could have antimicrobial, anti-inflammatory, and anti-influenza virus effects $[9,10]$. The $C$. sinensis seeds are rich in polysaccharides with high viscosity [11] and are promising, as are moisturizing agents in cosmetics. Moreover, the leaf extract of $C$. sinensis could inhibit tyrosinase activity, which is closely related to pigmentation [12]. However, there are few reports regarding the effects of the $C$. sinensis fruit extract on the skin aging process. Hamauzu et al. showed that phenolic compounds from $C$. sinensis fruit have diphenylpicrylhydrazyl (DPPH) radical scavenging activity and can inhibit lipid oxidation by 2,2'-azobis-(amidinopropane) dihydrochloride (AAPH) [13]. In addition, Strek et al. showed that the extract of $C$. japonica, which belongs to the same genus as $C$. sinensis, inhibited the activities of gelatinase matrix-metalloproteases (MMP)-2 and -9 [14]. Thus, the possibility of the anti-aging potential of the C. sinensis extract is promising.

The aim of this study was to determine the anti-aging efficacy of the $C$. sinensis fruit extract. We tested whether the $C$. sinensis extracts inhibited collagenase and elastase activity, and whether it exhibited superoxide dismutase (SOD)-like activity. In addition, these activities were compared between the extracts of the whole fruit and the sarcocarp (seeds removed) in order to ascertain whether the effects were due to the polysaccharides in the seeds or the phenolic compounds in the sarcocarp.

\section{Materials and Methods}

\subsection{Plant Material}

The fruit of $C$. sinensis were obtained from Nara Prefectural Pharmaceutical Research Center (Nara, Japan).

\subsection{Reagents}

Trizma ${ }^{\circledR}$ base, Triton X-100, trypsin from bovine pancreas, and trypsin inhibitor from Glycine max (soybean) were purchased from Sigma-Aldrich Co., LLC. (St. Louis, MO, USA). Folin-Ciocalteu's phenol reagent was purchased from MP Biomedicals, LLC. (Santa Ana, CA, USA). (+)-Catechin and cyanidin chloride were purchased from Tokyo Chemical Industry Co., Ltd. (Tokyo, Japan). Procyanidin B1 and procyanidin B2 were purchased from EXTRASYNTHESE (Genay Cedex, France). N-methoxysuccinyl-L-alanyl-L-alanyl-L-prolyl-L-valine 4-methylcoumaryl-7-amide (MeO-Suc-Ala-Ala-Pro-Val-MCA) was purchased from Peptide Institute (Osaka, Japan). Ethylenediamine- $N, N, N^{\prime}, N^{\prime}$-tetraacetic acid disodium salt dehydrate (EDTA) and o-phenanthroline were purchased from Dojindo Laboratories (Kumamoto, Japan). Human neutrophil elastase (HNE: EC 3.4.21.37) and xanthine oxidase from buttermilk (EC 1.1.3.22) were purchased from Merck (Darmstadt, Germany). Collagenase from Clostridium histolyticum and fluorescein isothiocyanate (FITC)-labeled bovine type I collagen were obtained from Worthington Biochemical Corp. (Lakewood, NJ, USA) and the Collagen Research Center (Tokyo, Japan), respectively. All the other chemicals used were purchased from FUJIFILM Wako Pure Chemical Corp. (Osaka, Japan).

\subsection{Extracts Preparation}

C. sinensis extracts were prepared from two forms of the fruit: the whole fruit and sarcocarps (in which the seeds were removed from whole fruit). Whole fruit and sarcocarps of $C$. sinensis were dried, powdered, and extracted with water at $100{ }^{\circ} \mathrm{C}$ for $30 \mathrm{~min}$ under reflux. The resulting extracts were filtered (300 mesh gauze) and lyophilized to remove the solvent. 


\subsection{Isolation of Compounds from an Extract of C. sinensis sarcocarp}

The extract of the $C$. sinensis sarcocarp was chromatographed using Diaion HP20 (Mitsubishi Chemical Corp., Tokyo, Japan) and successively eluted with water (Fr.1), 30\% methanol (Fr.2), 50\% methanol (Fr.3), 60\% methanol (Fr.4), 80\% methanol (Fr.5), and 100\% methanol (Fr.6). To remove the solvent, the resulting fractions were lyophilized or evaporated or both.

\subsection{Elastase Inhibition Assay}

The elastase inhibition assay was performed as described previously [15]. The reaction mixture contained the extracts, $0.2 \mathrm{M}$ Tris- $\mathrm{HCl}$ buffer $(\mathrm{pH} 8.5), 1 \mu \mathrm{g} / \mathrm{mL} \mathrm{HNE}$, and $25 \mu \mathrm{M}$ MeO-Suc-Ala-Ala-Pro-Val-MCA. The mixtures were incubated at $37^{\circ} \mathrm{C}$ for $60 \mathrm{~min}$, and fluorescence was measured at $365 \mathrm{~nm}$ excitation and 410-460 nm emission using a 96-well microplate reader (Glomax ${ }^{\circledR}$-Multi Detection System, Promega, Tokyo, Japan). The percentage inhibition for this assay was calculated as follows:

$$
\text { Elastase inhibition }(\%)=\{[(\mathrm{A}-\mathrm{B})-(\mathrm{C}-\mathrm{D})] /(\mathrm{A}-\mathrm{B})\} \times 100
$$

where $\mathrm{A}$ is the fluorescence intensity without the extracts (control), $\mathrm{B}$ is the fluorescence intensity without the extracts and enzyme (blank of A), C is the fluorescence intensity with the extracts, and D is the fluorescence intensity with the extracts and without the enzyme. Phenylmethylsulfonyl fluoride (PMSF; FUJIFILM Wako Pure Chemical Corp.) was used as a positive control in this assay.

\subsection{Assay for SOD-Like Activity}

Assay for SOD-like activity was performed as described previously [15]. The reaction mixture contained the extracts, $0.2 \mathrm{M}$ Tris- $\mathrm{HCl}$ buffer (pH 7.8), $0.1 \mathrm{mM}$ EDTA-2Na, $0.1 \mathrm{mM}$ xanthine, $0.05 \mathrm{mM}$ nitro blue tetrazolium, and $0.05 \mathrm{U}$ xanthine oxidase. The mixture was incubated at $37{ }^{\circ} \mathrm{C}$ for $25 \mathrm{~min}$ and absorbance was measured at $560 \mathrm{~nm}$ using a U-3010 UV/vis spectrophotometer (Hitachi High-Technologies Co., Ltd., Tokyo, Japan). SOD-like activity was calculated as follows:

$$
\text { SOD-like activity }(\%)=\{[(\mathrm{A}-\mathrm{B})-(\mathrm{C}-\mathrm{D})] /(\mathrm{A}-\mathrm{B})\} \times 100
$$

where $\mathrm{A}$ is the absorbance without the extracts (control), $\mathrm{B}$ is the absorbance without the extracts and enzyme (blank of A), C is the absorbance with the extracts, and D is the absorbance with the extracts and without enzyme. SOD (human, recombinant; FUJIFILM Wako Pure Chemical Corp.) was used as a positive control in this assay.

\subsection{Assay of Inhibition of Collagenase from Clostridium histolyticum}

The collagenase inhibition assay was performed, as described previously [15]. The reaction mixture contained the extracts or Fr.1-6 samples, $0.025 \mathrm{M}$ Tris- $\mathrm{HCl}$ buffer (pH 7.5), $0.25 \mathrm{mg} / \mathrm{mL}$ FITC-labeled collagen, and $0.05 \mathrm{U}$ collagenase. Mixtures were incubated at $37{ }^{\circ} \mathrm{C}$ for $2 \mathrm{~h}$, and $o$-phenanthroline was then added $(2 \mathrm{mM})$ to stop the reaction. Extraction buffer $(70 \% \mathrm{EtOH}$ in $0.05 \mathrm{M}$ Tris- $\mathrm{HCl}, \mathrm{pH} 9.5$ ) was added, and the mixtures were centrifuged at $1750 \times g$ for $10 \mathrm{~min}$. Fluorescence was measured in the supernatants at $490 \mathrm{~nm}$ excitation and 510-570 $\mathrm{nm}$ emission wavelengths using a 96-well microplate reader (Glomax ${ }^{\circledR}$-Multi Detection System). The percentage inhibition for this assay was calculated as follows:

$$
\text { Collagenase inhibition }(\%)=\{[(\mathrm{A}-\mathrm{B})-(\mathrm{C}-\mathrm{D})] /(\mathrm{A}-\mathrm{B})\} \times 100
$$

where $\mathrm{A}$ is the fluorescence intensity without the samples (control), B is the fluorescence intensity without the samples and enzyme (blank of A), C is the fluorescence intensity with the samples, and 
$\mathrm{D}$ is the fluorescence intensity with the samples and without enzyme. EDTA was used as a positive control in this assay.

\subsection{D Human Skin Model and Assay for Collagenase Activity}

EpiDerm ${ }^{\mathrm{FT}}$ full-thickness reconstituted skin tissue (EFT-400, MatTek Corp., Ashland, MA, USA) was a multilayered model of human epidermis and dermis. EFT- 400 was pretreated with the sarcocarp extract for $16-18 \mathrm{~h}$ at $37^{\circ} \mathrm{C}$ in a humidified atmosphere $\left(5 \% \mathrm{CO}_{2}\right)$. The sample was removed, and then EFT-400 was irradiated with NTM-20 transilluminator (UVP, CA, USA) at $365 \mathrm{~nm}$. After UV irradiation, EFT- 400 was re-treated with the sample for $24 \mathrm{~h}$ at $37^{\circ} \mathrm{C}$ in a humidified atmosphere (5\% $\left.\mathrm{CO}_{2}\right)$. EFT-400 was lysed in a lysis buffer $\left(0.2 \mathrm{M} \mathrm{NaCl}, 5 \mathrm{mM} \mathrm{CaCl}_{2}, 0.1 \%\right.$ Triton X-100, pH 7.4). The lysate was centrifuged at $1300 \times g$ for $10 \mathrm{~min}$. Ten microliters of trypsin $(4 \mathrm{mg} / \mathrm{mL})$ was added to $80 \mu \mathrm{L}$ of the supernatant and incubated for $5 \mathrm{~min}$ at $37^{\circ} \mathrm{C}$. Next, $10 \mu \mathrm{L}$ of trypsin inhibitor $(20 \mathrm{mg} / \mathrm{mL})$ was added. The resultant solution was used in the assay for collagenase activity. The reaction mixture contained the lysate solution, $0.025 \mathrm{M}$ Tris- $\mathrm{HCl}$ buffer ( $\mathrm{pH} 7.5$ ), and $0.25 \mathrm{mg} / \mathrm{mL}$ FITC-labeled collagen. Mixtures were incubated at $37^{\circ} \mathrm{C}$ for $2 \mathrm{~h}$, followed by the addition of $o$-phenanthroline $(2 \mathrm{mM})$ to stop the reaction. Extraction buffer $(70 \% \mathrm{EtOH}$ in $0.05 \mathrm{M}$ Tris- $\mathrm{HCl}, \mathrm{pH} 9.5)$ was added, and the mixtures were centrifuged at $1750 \times g$ for $10 \mathrm{~min}$. Fluorescence of the supernatants was measured at $490 \mathrm{~nm}$ excitation and $510-570 \mathrm{~nm}$ emission wavelengths using a 96-well microplate reader (Glomax ${ }^{\circledR}$-Multi Detection System). The percentage collagenase activity for this assay was calculated as follows:

$$
\text { Collagenase activity }(\%)=\{(\mathrm{C}-\mathrm{B}) /(\mathrm{A}-\mathrm{B})\} \times 100
$$

where A is the fluorescence intensity of non-treat cell lysate (control), B is the fluorescence intensity without cell lysate (blank of A), and C is the fluorescence intensity of cell lysate treated with UV irradiation or the sample or both.

\subsection{3-(4,5-Dimethylthiazol-2-yl)-2,5-Diphenyltetrazolium Bromide (MTT) Assay}

EFT-400 was treated with UV irradiation or the sarcocarp extract or both and was placed in $1.5 \mathrm{~mL}$ tube containing $2.5 \mathrm{mg} / \mathrm{mL}$ MTT solution. After $3 \mathrm{~h}$ of incubation at $37^{\circ} \mathrm{C}$ in a humidified atmosphere $\left(5 \% \mathrm{CO}_{2}\right)$, EFT-400 was immersed in an extraction solution (isopropyl alcohol), and then incubated overnight at $4{ }^{\circ} \mathrm{C}$. After overnight extraction, the absorbance was measured at $570 \mathrm{~nm}$ using a 96-well microplate reader (iMark ${ }^{\mathrm{TM}}$ Microplate Reader, Bio-Rad Laboratories Inc., Hercules, CA, USA). The percentage viability was calculated as follows:

$$
\text { Viability }(\%)=\{(\mathrm{C}-\mathrm{B}) /(\mathrm{A}-\mathrm{B})\} \times 100
$$

where A is the absorbance intensity of non-treat cell lysate (control), B is the absorbance intensity without cell lysate (blank of A), and C is the absorbance intensity of cell lysate treated with UV irradiation or the sample or both.

\subsection{Total Phenolic Content}

The total phenolic content was determined in accordance with our previous study [15], which uses the Folin-Ciocalteu reagent. The reaction mixture contained the Fr.1-6 samples, $0.5 \mathrm{~N}$ Folin-Ciocalteu reagent, and $0.29 \mathrm{M}$ sodium carbonate. After the mixture was incubated at $50{ }^{\circ} \mathrm{C}$ for $5 \mathrm{~min}$, the absorbance at $765 \mathrm{~nm}$ was measured using a U-2000 spectrophotometer (Hitachi High-Technologies Co., Ltd., Tokyo, Japan). The total phenolic content was calculated from standard curves of absorbance against the concentration of standard gallic acid monohydrate solutions and expressed as gallic acid monohydrate equivalents. 


\subsection{Total Condensed Tannin Content}

The Fr.1-6 samples $(500 \mu \mathrm{L})$ were mixed with $3 \mathrm{~mL}$ of vanillin reagent ( $4 \%$ vanillin in methanol) and $1.5 \mathrm{~mL}$ sulfuric acid. The mixture was incubated at room temperature (from 20 to $25^{\circ} \mathrm{C}$ ) for $15 \mathrm{~min}$, and then the absorbance at $500 \mathrm{~nm}$ was measured using a U-3010 UV/vis spectrophotometer (Hitachi High-Technologies Co., Ltd.). The condensed tannin content was calculated from standard curves of absorbance against the concentration of standard catechin solutions and expressed as catechin equivalents.

\subsection{Sample Acetylation and Gel Permeation Chromatography (GPC)}

The Fr.3 sample was acetylated overnight at room temperature (from 20 to $25^{\circ} \mathrm{C}$ ) with acetic anhydride/pyridine (1:1) solution. After completion of the reaction, the acetylated sample was precipitated by the addition of water, and then centrifuged at $2000 \times g$ for $10 \mathrm{~min}$. The precipitation was dried under nitrogen and then dissolved in tetrahydrofran (THF). This solution was used in GPC analysis. The analysis of molecular size was performed by GPC analysis using a Waters 2690 separation module (Waters Co., Milford, MA, USA) coupled to a Waters 996 photodiode array detector (Waters Co.). Separation was conducted using Inertsil ${ }^{\circledR}$ WP300 Diol column $(5 \mu \mathrm{m}, 4.6 \times 250 \mathrm{~mm}$, GL Science, Inc., Tokyo, Japan) with a Cartridge Guard Column E Inertsil ${ }^{\circledR}$ WP300 Diol (5 $\mu \mathrm{m}, 4.0 \times 10 \mathrm{~mm}$, GL Science, Inc.). The mobile phase was THF, with a flow rate of $0.35 \mathrm{~mL} / \mathrm{min}$ and a column temperature of $40{ }^{\circ} \mathrm{C}$. UV detection was performed at $254 \mathrm{~nm}$. Polystyrene standards (Shodex Standard SM-105, Showa Denko KK, Tokyo, Japan) were used to calibrate standards for GPC. The mean polymerization degree of the sample was calculated as follows:

$$
\text { Mean polymerization degree }=\mathrm{A} / \mathrm{B}
$$

where A is the peak-top molecular size from GPC, and B is the molecular weight of catechin $(\mathrm{MW}=290.27)$.

\subsection{Decomposition and Analysis of Phenolic Composition of Sample Using High Performance Liquid Chromatography (HPLC) Analysis}

Phenolic composition of the Fr.3 sample was analyzed using HPLC. HPLC analysis was carried out on a Waters 2690 separation module coupled to a Waters 996 photodiode array detector. Separation

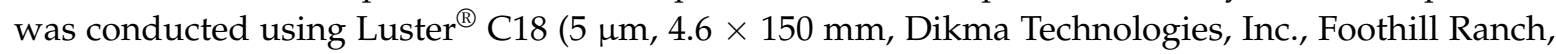
CA, USA). Solvents used for the analysis of polyphenols were $0.1 \%$ phosphoric acid (A) and $0.1 \%$ phosphoric acid in acetonitrile (B). The gradient program was started with $5 \% \mathrm{~B}$ and then changed to obtain 15\% B at $30 \mathrm{~min}, 32 \% \mathrm{~B}$ at $35 \mathrm{~min}$, and $36 \% \mathrm{~B}$ at $40 \mathrm{~min}$, with a flow rate of $1 \mathrm{~mL} / \mathrm{min}$ and a column temperature of $40{ }^{\circ} \mathrm{C}$. UV detection was performed at $280 \mathrm{~nm}$.

Decomposition of sample was conducted using hydrochloric acid solution. The Fr.3 sample was acid treated with $30 \%$ hydrochloric acid solution at $95^{\circ} \mathrm{C}$ for $2 \mathrm{~h}$. After reaction, the acid sample was stirred with the addition of acetonitrile (final concentration; $17 \%$ ), and then filtered at $0.2 \mu \mathrm{m}$. HPLC analysis was carried out on a Waters 2690 separation module coupled to a Waters 996 photodiode array detector. Separation was conducted using Luster ${ }^{\circledR}$ C18 (5 $\mu \mathrm{m}, 4.6 \times 150 \mathrm{~mm}$, Dikma Technologies, Inc.). Solvents used for analysis of polyphenols were $4 \%$ phosphoric acid (A) and acetonitrile (B). The gradient program was initiated with $10 \% \mathrm{~B}$ and then changed to obtain $17 \% \mathrm{~B}$ at $15 \mathrm{~min}$, with a flow rate of $1 \mathrm{~mL} / \mathrm{min}$ and a column temperature of $35^{\circ} \mathrm{C}$. UV detection was performed at $280 \mathrm{~nm}$.

\subsection{Statistical Analysis}

The experimental data was evaluated for statistical significance using Student's t-test. A value of $p<0.05$ was considered statistically significant. 


\section{Results}

\subsection{Extract Preparation}

Extracts from the $C$. sinensis were prepared with water. When the extract from $C$. sinensis was prepared and filtered through a mesh, the extract of the whole fruit showed an increased viscosity as the extraction temperature decreased. After removing the solvent, the extract was weighed and the yield was calculated. The results are expressed in $\mathrm{mg} / \mathrm{g}$. The yield of the sarcocarps $(32.5 \pm 9.4, \mathrm{n}=7)$ was higher than that of the whole fruit $(16.0 \pm 0.8, \mathrm{n}=3)$.

\subsection{Analysis of Elastase Inhibition Effect Using Human Neutrophil Elastase}

Extracts were tested for inhibitory effects on elastase using human neutrophil elastase. For the positive control, the known elastase activity inhibitor PMSF, which has serine at the active center, was used. PMSF decreased enzymatic activity in a concentration-dependent manner. Both sarcocarps and whole fruit showed elastase inhibition, with the whole fruit extracts $(80 \%$ inhibitory concentration; IC $80=0.022 \%$ ) showing better effect than that of the sarcocarps (seeds removed, IC80 $>0.05 \%$ ) (Figure 1). It was revealed that the seeds contained many components with inhibitory effects on elastase.

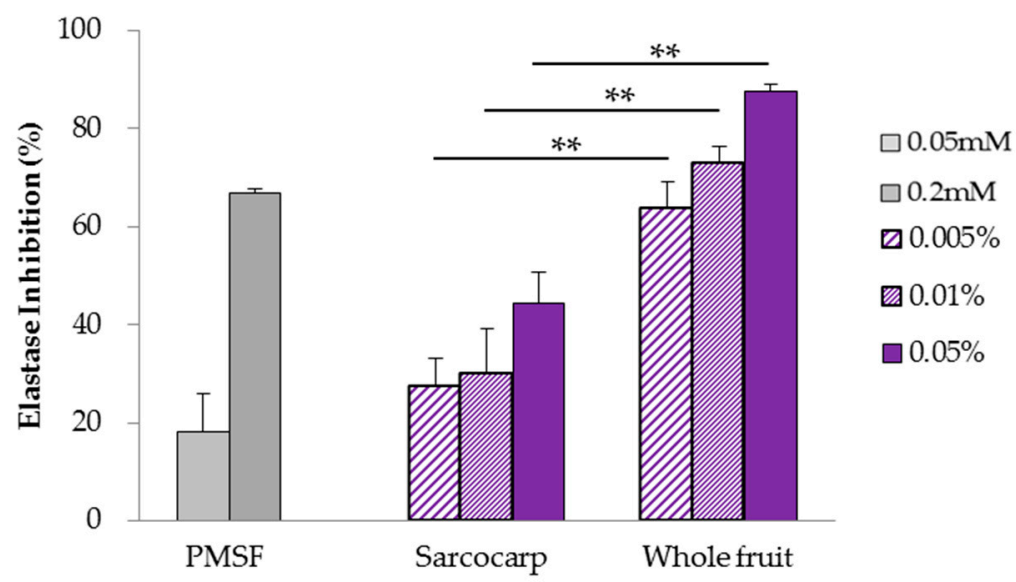

Figure 1. Elastase inhibition effect of the aqueous extracts from C. sinensis. Equal quantities of sarcocarp and whole fruit extracts were dissolved in the buffer to the appropriate concentration, and the elastase inhibition was determined. The sample concentration is described as the solid concentration. Results are expressed as the mean $\pm \mathrm{SD}(\mathrm{n}=3)(* * p<0.01)$.

\subsection{Analysis of SOD-Like Activity Using Xanthine Oxidase}

Extracts were tested for SOD-like activity using xanthine oxidase. SOD was used as a positive control, and its activity increased in a concentration-dependent manner. The $C$. sinensis sarcocarps $(\mathrm{IC} 80=0.0039 \%)$ had higher SOD-like activity than the whole fruit $(\mathrm{IC} 80>0.01 \%)(p<0.01)($ Figure 2$)$. It was revealed that the sarcocarps, not the seeds, contained ingredients with SOD-like activity. 


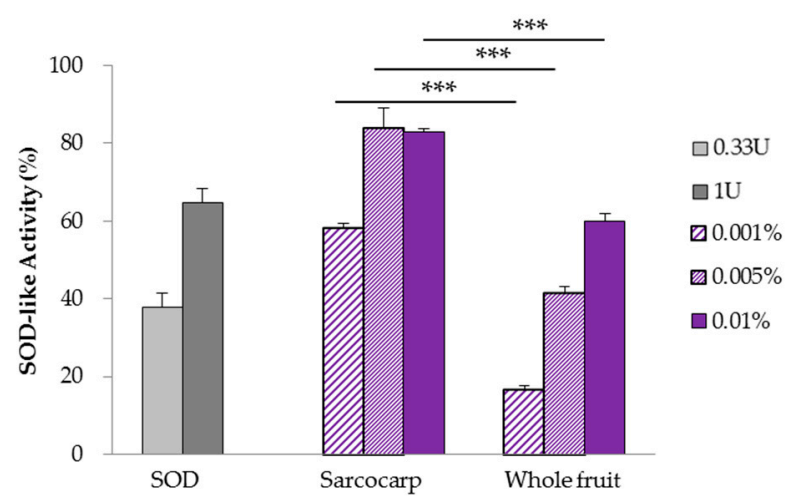

Figure 2. SOD-like activity in the aqueous extracts from $C$. sinensis. Equal quantities of sarcocarp and whole fruit extracts were dissolved in distilled water to the appropriate concentration, and the SOD-like activity was determined. The sample concentration is described as the solid concentration. Results are expressed as the mean $\pm \operatorname{SD}(\mathrm{n}=3)\left({ }^{* * *} p<0.001\right)$.

\subsection{Analysis of Collagenase Inhibition Effect Using Clostridium histolyticum Collagenase}

Extracts were tested for inhibitory effects on collagenase using collagenase from Clostridium histolyticum. EDTA is a well-known collagenase inhibitor and decreases the enzymatic activity in a concentration-dependent manner. The inhibitory effect of the sarcocarps (IC $80=0.0022 \%$ ) on collagenase was greater than that of the whole fruit (IC $80=0.21 \%$ ) (Figure 3). It was revealed that the sarcocarps, not the seeds, contained components with inhibitory effects on collagenase.

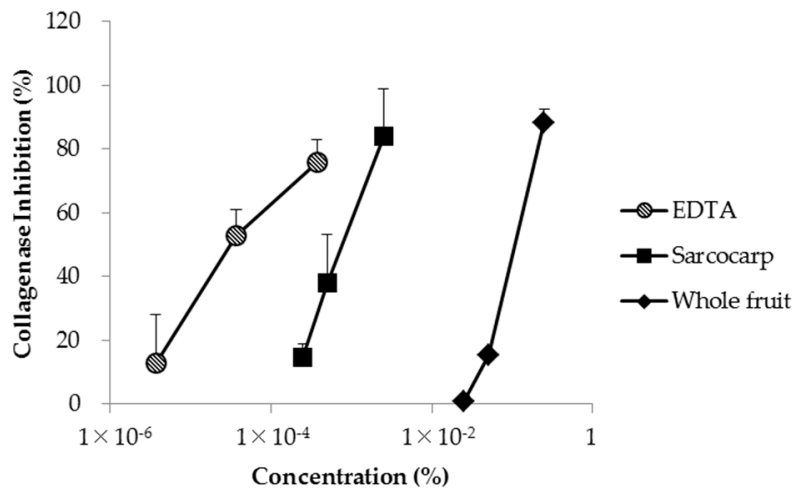

Figure 3. Collagenase inhibition effect of the aqueous extracts from C. sinensis. Equal quantities of sarcocarp and whole fruit extracts were dissolved in distilled water to the appropriate concentration, and the collagenase inhibition was determined. The sample concentration is described as the solid concentration. Results are expressed as the mean \pm SD $(n=3-6)$.

\subsection{Analysis of Inhibition Effect on Collagenase Activity Induced by UV Irradiation}

The UV exposure activates skin collagenase often via the activation of neutrophil elastase and ROS generation. The extracts of $C$. sinensis whole fruit and sarcocarps had inhibitory effects against elastase, collagenase, and SOD-like activity (Figures 1-3) in the enzyme reaction assays in cell-free systems. In particular, the sarcocarp extract showed high SOD-like activity as well as a considerable inhibitory effect on collagenase at a low concentration. Therefore, we used a human skin model and evaluated the effect of the sarcocarp extract on collagenase activity induced by UV irradiation. The experiment was designed to characterize the cell viability and collagenase activity of the human skin model exposed to UV irradiation $\left(60 \mathrm{~J} / \mathrm{cm}^{2}\right)$. Compared to the untreated human skin model, the collagenase activity of the UV-irradiated human skin model (without $C$. sinensis sarcocarps) was enhanced. In contrast, the collagenase activity of the UV-irradiated skin model treated with the C. sinensis sarcocarps showed a decline compared to that not treated with C. sinensis (Figure 4a). The viability of the skin models, 
which were UV-irradiated or treated with C. sinensis or both, was not reduced. (Figure $4 b$ ). In addition to inhibition of collagenase derived from Clostridium histolyticum, it was revealed that the sarcocarps contained components that inhibited collagenase activity by UV irradiation.
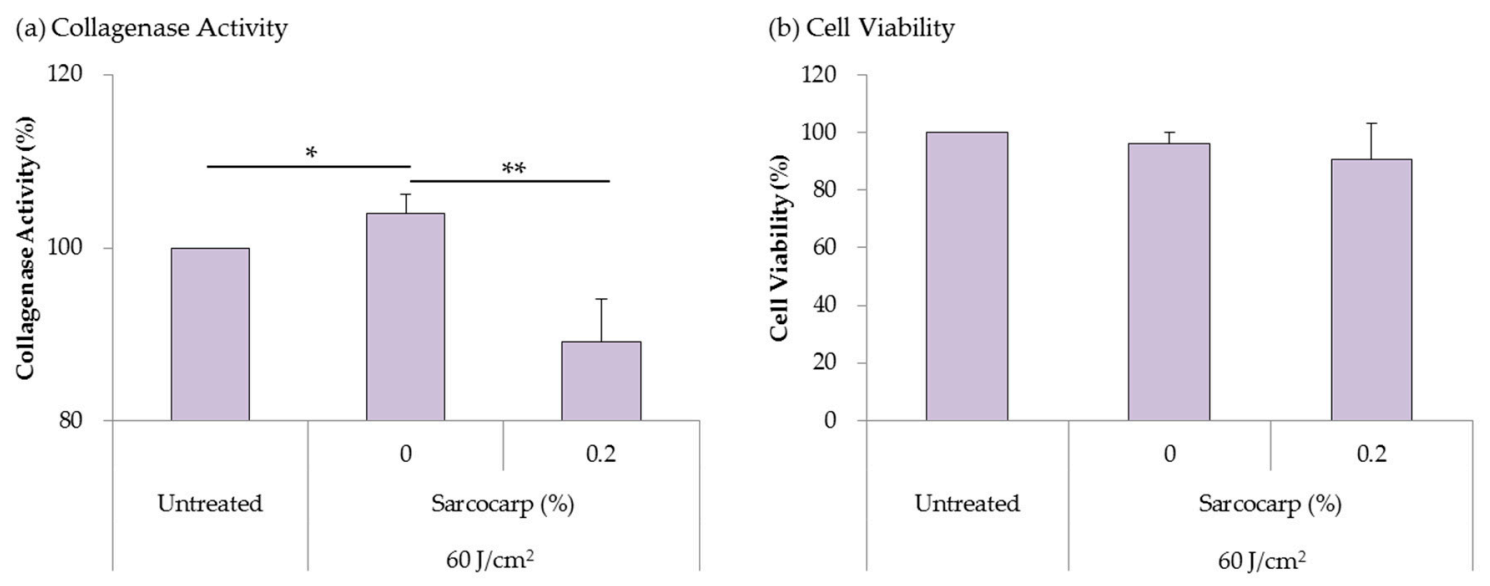

Figure 4. The human skin model was irradiated with UV. (a) The changes in collagenase activity. (b) The human skin model viability. The sample concentration is described as the solid concentration. Results are expressed as the mean $\pm \mathrm{SD}(\mathrm{n}=3)\left({ }^{*} p<0.05,{ }^{* *} p<0.01\right)$.

\subsection{Total Phenolic and Condensed Tannin Content}

In order to analyze the main active components, the extracts of the $\mathrm{C}$. sinensis sarcocarps were fractionated (Fr.1-Fr.6) using column chromatography. Fr.2-5 showed the higher phenolic content than sarcocarp (168). Fr.3 (1004) showed the highest phenolic content among all the fractions, followed by Fr.2 (626), Fr.4 (505), Fr.5 (362), Fr.6 (17), and Fr.1 (13). Fr.3 (1004) showed the highest phenolic content amongst all the fractions, followed by Fr.2 (626), Fr.4 (505), Fr.5 (362), Fr.6 (17), and Fr.1 (13) (Table 1).

Table 1. The total phenolic content.

\begin{tabular}{ccc}
\hline Fraction & Total Phenolic Content (mg/g extract) & Total Condensed Tannin Content (mg/g Extract) \\
\hline Sarcocarp & $168 \pm 5$ & $150 \pm 10$ \\
Fr.1 & $13 \pm 2$ & $<25$ \\
Fr.2 & $626 \pm 14$ & $397 \pm 29$ \\
Fr.3 & $1004 \pm 38$ & $688 \pm 103$ \\
Fr.4 & $505 \pm 19$ & $205 \pm 10$ \\
Fr.5 & $362 \pm 38$ & $64 \pm 4$ \\
Fr.6 & $17 \pm 1$ & $<25$ \\
\hline
\end{tabular}

The total condensed tannin content was measured using the phenol-sulfuric acid method. Condensed tannins were determined in comparison with the absorbance values (obtained in milligrams) of catechin per gram. Fr.2-4 showed the higher total condensed tannin content than sarcocarp (150). Fr.3 (688) showed the highest tannin content among all the fractions, followed by Fr.2 (397), Fr.4 (205), Fr.5 (64), Fr.1 (<25), and Fr.6 (<25) (Table 1).

\subsection{Analysis of Collagenase Inhibition Effect Using Clostridium histolyticum Collagenase}

In order to analyze the active components in the C. sinensis sarcocarps, the inhibitory effects on collagenase exerted by the sarcocarps and the isolated fractions (Fr.1-6) from the sarcocarps were compared. The collagenase inhibition exerted by the samples was measured by the fluorescence method using Clostridium histolyticum collagenase. The sample concentration was described as sarcocarps equivalent, calculated by comparing the response rate in each fraction. Fr. 3 showed the highest inhibitory effect in comparison with the other fractions, followed by Fr.2. In contrast, Fr.1, Fr.4, 
Fr.5, and Fr.6 showed no collagenase inhibition (Figure 5). The activity of Fr.2 and Fr.3 was close to that of the sarcocarps before fractionation. Further, the activity of Fr.3 was much higher than that of Fr.2. It was revealed that the main active component was present in Fr.3.

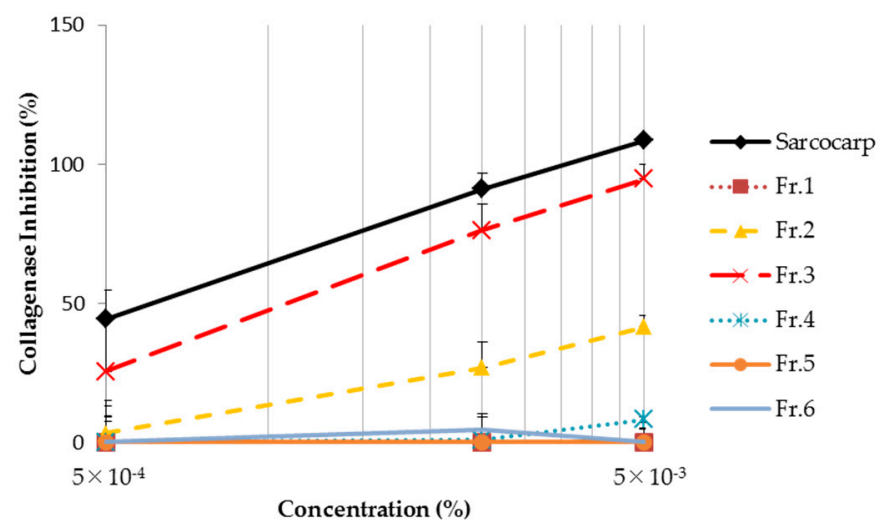

Figure 5. Collagenase inhibition effect of the fractions obtained from the extract of $C$. sinensis sarcocarp. The sarcocarp extract and fractions were dissolved in distilled water to the appropriate concentration, and the collagenase inhibition was determined. Results are expressed as the mean \pm SD $(n=3)$. The sample concentration is described as the sarcocarp equivalent, calculated by the response rate in each fraction.

\subsection{Analysis of Polymeric Polyphenols in Fr.3}

Fr.3 was rich in phenolic compounds and condensed tannins and showed high collagenase inhibition (Table 1, Figure 5). The phenolic composition of Fr.3 was analyzed using HPLC. Peaks were characterized by comparing their retention times and UV-spectra with standards. Procyanidin B2 was identified and probable polymeric procyanidins appeared as unresolved, slightly sharp clumps for Fr.3 (Figure 6a). Analysis of the decomposed components of Fr.3 by acid treatment followed by HPLC showed a peak coinciding with the peak of cyanidin chloride (Figure 6b). These results indicated that main phenolic compounds in Fr.3 were cyanidin polymers. The analysis of molecular size was performed by GPC analysis. The peak-top molecular weight of Fr.3 was $1617 \mathrm{~g} / \mathrm{mol}$, and the average polymerization degree (calculated by molecular weight of catechin) of the sample was 5.57.

(a) Before treatment

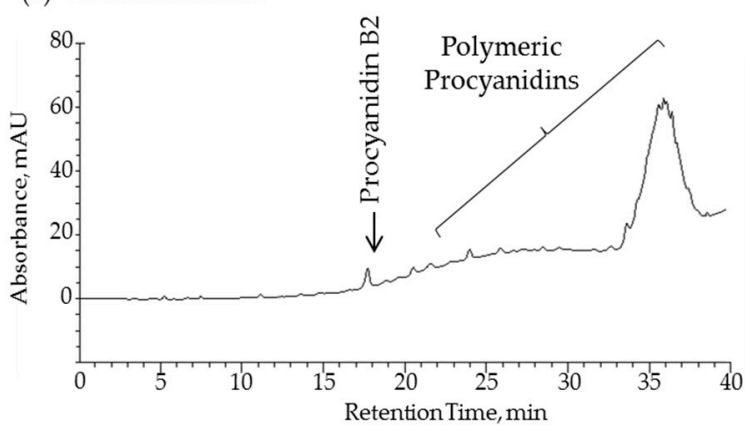

(b) Acid treatment

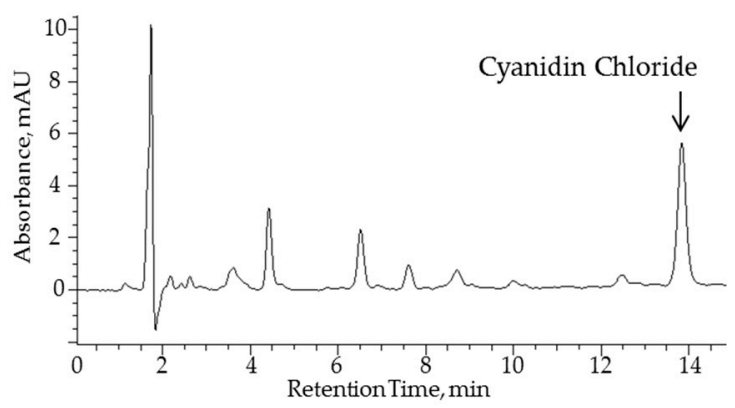

Figure 6. HPLC chromatograms obtained on a Luster ${ }^{\circledR} \mathrm{C} 18(5 \mu \mathrm{m}, 4.6 \times 150 \mathrm{~mm})$ column at $280 \mathrm{~nm}$. Peaks have been characterized according to their retention times and UV-spectra. (a) Chromatogram of Fr.3 before acid treatment. (b) Chromatogram of decomposed components of Fr.3 after acid treatment.

Polymeric procyanidins mainly consist of (+)-catechin and (-)-epicatechin units, which are flavan-3-ol monomers. Fr.3 contained cyanidin dimer, procyanidin B2, and higher polymerization cyanidins. The inhibitory effect on collagenase exerted by monomers ((+)-catechin, (-)-epicatechin), dimers (procyanidin B1 and B2), and Fr.3 were compared. It was observed that procyanidin B1 and B2 
showed higher collagenase inhibition than (+)-catechin and (-)-epicatechin, and Fr.3 showed greater activity than procyanidin B1 and B2 (Figure 7).

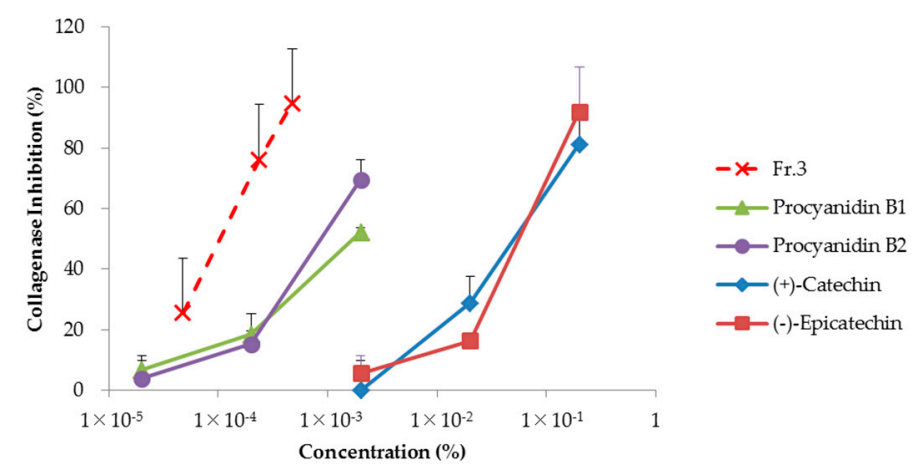

Figure 7. Collagenase inhibition effect of sarcocarps Fr.3, catechins, and procyanidins. Samples were dissolved in distilled water to the appropriate concentration, and the collagenase inhibition was determined. The sample concentration is described as the solid concentration. Results are expressed as the mean $\pm S D(n=3-4)$.

\section{Discussion}

Skin aging is a complex process caused by various intrinsic and extrinsic factors. Intrinsic aging is caused by the increase in chronological age, and extrinsic factors such as UV irradiation are known to accelerate the intrinsic aging process. In normal skin, collagen and elastin are produced and decomposed repeatedly in order to maintain the dermal structure. However, UV exposure causes a decrease in collagen and elastin fibers in the connective tissue of the skin. These fibers are degraded by MMP or serine proteinase. Neutrophil elastase, a serine proteinase, is secreted by neutrophils in the acute inflammation phase after UV irradiation. It has been reported that neutrophil elastase is indirectly involved in collagen and elastin degradation through MMP-1 and MMP-2 activation as well as by direct degradation [5]. It has also been reported that exposure to UV and particulate matter 2.5 (PM 2.5) induces excessive ROS generation, leading to DNA damage in normal cells and increased MMP (collagenase) levels, which can promote skin aging [15-18]. It is considered that supplements that prevent elastase and collagenase activity, as well as superoxide generation, are useful as ingredients in cosmetics that slow down skin aging. In this study, we determined the anti-aging potential of $C$. sinensis fruit extracts using assays to evaluate their inhibitory effects on these proteases and superoxide dismutase. In addition, the inhibitory effects of the different extracts from the whole fruit and sarcocarps (seeds removed) were also compared.

In the elastase inhibition assay, the $C$. sinensis extract inhibited neutrophil elastase activity, and the extract of the whole fruit $(80 \%$ inhibitory concentration; IC $80=0.022 \%$ ) was more effective than that of the sarcocarp (seeds removed, IC $80>0.05 \%$ ) (Figure 1). This means that the components in the seeds had a greater inhibitory effect on elastase than those in the sarcocarp. It is known that the $C$. sinensis seeds are rich in viscous, acidic polysaccharides, which include a $\beta-1,4$-xyloglucan main-chain and a side-chain connected to arabinose, glucose, and glucuronic acid [11]. As the whole fruit extract used in this study was viscous, we considered that the extract contained acidic polysaccharides. It has been reported that polysaccharides extracted from Kochia scoparia inhibited neutrophil elastase activity [19]. Hence, it is likely that polysaccharides in C. sinensis seeds inhibited the elastase activity.

For the SOD-like activity assay, the extract of the sarcocarp exhibited higher activity $(\mathrm{IC} 80=0.0039 \%)$ than the whole fruit extract (IC80 > 0.01\%) (Figure 2), in contrast to the elastase activity. The total phenolic content of the sarcocarp extract was four times higher than the whole fruit extract, and most of the phenolic compounds were condensed tannins (data not shown). Phenolic compounds such as catechin, epicatechin, and procyanidin B1 and B2 extracted from various plants have been reported to exhibit SOD-like activity $[20,21]$. Procyanidin belongs to the flavonoid class 
of flavan-3-ol and exists as an oligomer or polymer ( 2 to 15 mer) to which catechin or epicatechin is condensed [22]. Furthermore, dimeric and trimeric procyanidins have stronger antioxidant activity than monomeric catechins [23]. It was suggested that the condensed tannins in the $C$. sinensis sarcocarp were responsible for the SOD-like activity.

For the collagenase inhibition assay, the extract of the sarcocarp (IC80 $=0.0022 \%$ ) inhibited collagenase from Clostridium histolyticum at a much lower concentration than that of the whole fruit extract (IC80 $=0.21 \%$ ) (Figure 3). Though the extract of the whole fruit showed a greater inhibitory effect on elastase $(\mathrm{IC} 80=0.022 \%)$ than that of the sarcocarp, a lower concentration of the sarcocarp extract showed a high SOD-like activity (IC $80=0.0039 \%$ ) as well as a considerable inhibitory effect on collagenase (IC80 $=0.0022 \%$ ) (Figures 1-3). Therefore, we focused on the sarcocarp extract and investigated its effect on the collagenase activity induced by UV irradiation, using a full-thickness reconstituted skin tissue. As Figure 4 shows, the sarcocarp extract inhibited the collagenase activity induced by UVA. Thus, because the $C$. sinensis sarcocarp extract also showed inhibitory effects against neutrophil elastase and superoxide generation (Figures 1 and 2), it was considered that the $C$. sinensis sarcocarp indirectly inhibited the collagenase activity via the inhibition of neutrophil elastase and superoxide generation. However, the results also suggested the presence of a particular constituent in the $C$. sinensis sarcocarp extract that was directly affecting collagenase because a much lower concentration of the extract was needed to inhibit the Clostridium histolyticum collagenase activity than that required against the neutrophil elastase activity and superoxide generation. Accordingly, we analyzed the active ingredients in the $C$. sinensis sarcocarp. Fr.3, which was prepared using HP20 from the extract of sarcocarp, was rich in phenolic compounds and condensed tannin, and showed superior collagenase inhibition (Figure 5 and Table 1) compared to the other fractions. Further, it was observed that Fr.3 mainly contained polymeric procyanidins (Figure $6 \mathrm{a}, \mathrm{b}$ ), and the peak-top polymerization degree was 5.57. Therefore, the inhibitory effects on collagenase exerted by the cyanidin monomers, dimers, and Fr.3 were compared. As a result, Fr.3 showed the highest activity, followed by the dimers and, finally, the monomers (Figure 7). It has been reported that green tea catechin inhibited collagenase activity [24]. These reports corresponded with our study. Furthermore, our result showed that the cyanidin polymer, which had higher polymerization degree, and also had higher inhibitory effects on collagenase. Therefore, it was suggested that the main constituents involved in collagenase inhibition were condensed tannins in Fr.3. It has been reported that the flowers and leaves of some Rosaceae plants have inhibitory effects on collagenase [25]. Moreover, to the best of our knowledge, there are very few reports of this activity in Rosaceae fruits. In this study, we found through in vitro assays that the extract of $C$. sinensis sarcocarp has a high collagenase inhibitory ability. In real human skin, it is considered that the active ingredients may be degraded by many enzymes. Moreover, active ingredients of the extract of $C$. sinensis, being polymeric components, may exhibit poor penetration of the dermis. However, in the full-thickness reconstituted skin tissue, which is similar to the structure of the human skin, the extract of $C$. sinensis sarcocarp inhibited collagenase activity when applied on the stratum corneum side, indicating that the active ingredients penetrated the skin tissue. Therefore, it was considered that the active ingredients penetrated to the skin tissue and the effect was exhibited. Thus, our findings in this study could provide new insights into the application of Rosaceae fruits as cosmetic ingredients.

Author Contributions: Conceptualization, S.I., M.Y., and K.S.; formal analysis, S.I., M.Y., and K.S.; investigation, S.I., M.Y., and K.S.; methodology, I.S.; project administration, I.S.; writing—original draft preparation, S.I., MY., I.S.

Funding: This research received no external funding.

Acknowledgments: We acknowledge Nara Prefectural Pharmaceutical Research Center (Nara, Japan) for plant materials used for experiments.

Conflicts of Interest: The authors declare no conflict of interest. 


\section{References}

1. Sveikata, K.; Balciuniene, I.; Tutkuviene, J. Factors influencing face aging. Literature review. Stomatologija 2011, 13, 113-116. [PubMed]

2. Brem, R.; Li, F.; Montaner, B.; Reelfs, O.; Karran, P. DNA breakage and cell cycle checkpoint abrogation induced by a therapeutic thiopurine and UVA radiation. Oncogene 2010, 29, 3953-3963. [CrossRef] [PubMed]

3. Rinnerthaler, M.; Bischof, J.; Streubel, M.K.; Trost, A.; Richter, K. Oxidative stress in aging human skin. Biomolecules 2015, 5, 545-589. [CrossRef] [PubMed]

4. Cavinato, M.; Waltenberger, B.; Baraldo, G.; Grade, C.V.C.; Stuppner, H.; Jansen-Dürr, P. Plant extracts and natural compounds used against UVB-induced photoaging. Biogerontology 2017, 18, 499-516. [CrossRef] [PubMed]

5. Takeuchi, H.; Gomi, T.; Shishido, M.; Watanabe, H.; Suenobu, N. Neutrophil elastase contributes to extracellular matrix damage induced by chronic low-dose UV irradiation in a hairless mouse photoaging model. J. Dermatol. Sci. 2010, 60, 151-158. [CrossRef] [PubMed]

6. Fisher, G.J.; Wang, Z.Q.; Datta, S.C.; Varani, J.; Kang, S.; Voorhees, J.J. Pathophysiology of premature skin aging induced by ultraviolet light. N. Engl. J. Med. 1997, 337, 1419-1428. [CrossRef]

7. Miyamoto, K. Characterization of Skin Photo-Aging through Skin Longitudinal Research, and It's Application to Skin-Aging Protection. J. JPN Cosmet. Sci. Soc. 2017, 41, 188-195.

8. e-Stat (Portal Site of Official Statistics of Japan). Research on the Production Trends of Specialty Fruit Trees. 2015. Available online: http:/ / www.webcitation.org/74e7cJhF6 (accessed on 14 December 2018).

9. Osawa, K.; Yasuda, H.; Morita, H.; Takeya, K.; Itokawa, H. Antibacterial and antihemolytic activity of triterpenes and $\beta$-Sitosterol isolated from Chinese Quince (Chaenomeles sinensis). Nat. Med. 1997, 51, 365-367.

10. Hamauzu, Y.; Yasui, H.; Inno, T.; Kume, C.; Omanyuda, M. Phenolic profile, antioxidant property, and anti-influenza viral activity of Chinese quince (Pseudocydonia sinensis Schneid.), quince (Cydonia oblonga Mill.), and apple (Malus domestica Mill.) fruits. J. Agric. Food Chem. 2005, 53, 928-934. [CrossRef]

11. Nakata, S.; Kakuta, M.; Misaki, A. Structure and functional properties of seed surface mucilage of karin (Chaenomelese sinensis). Trace Nutr. Res. 2009, 26, 46-49.

12. Matsuda, H.; Nakamura, S.; Kubo, M. Studies of cuticle drugs from natural sources. II. Inhibitory Effects of Prunus plants on melanin biosynthesis. Biol. Pharm. Bull. 1994, 17, 1417-1420. [CrossRef]

13. Hamauzu, Y.; Inno, T.; Kume, C.; Irie, M.; Hiramatsu, K. Antioxidant and antiulcerative properties of phenolics from Chinese quince, quince, and apple fruits. J. Agric. Food Chem. 2006, 54, 765-772. [CrossRef] [PubMed]

14. Strek, M.; Gorlach, S.; Podsedek, A.; Sosnowska, D.; Koziolkiewicz, M.; Hrabec, Z.; Hrabec, E. Procyanidin oligomers from Japanese quince (Chaenomeles japonica) fruit inhibit activity of MMP-2 and MMP-9 metalloproteinases. J. Agric. Food Chem. 2007, 55, 6447-6452. [CrossRef] [PubMed]

15. Yamaguchi, M.; Shigeyama, K.; Yoshikawa, M.; Matsumoto, T.; Yonezawa, Y.; Shiota, H.; Ueyama, T.; Sakaguchi, I. Effects of cultivation month and genetic background on phenolic content of Citrus tachibana peel. Biol. Pharm. Bull. 2018, 41, 1277-1281. [CrossRef] [PubMed]

16. Dupont, E.; Gomez, J.; Bilodeau, D. Beyond UV radiation: A skin under challenge. Int. J. Cosmet. Sci. 2013, 35, 224-232. [CrossRef] [PubMed]

17. Krutmann, J.; Bouloc, A.; Sore, G.; Bernard, B.A.; Passeron, T. The skin aging exposome. J. Dermatol. Sci. 2017, 85, 152-161. [CrossRef]

18. Verma, V.; Fang, T.; Guo, H.; King, L.; Bates, J.T.; Peltier, R.E.; Edgerton, E.; Russell, A.G.; Weber, R.J. Reactive oxygen species associated with water-soluble PM2.5 in the southeastern United States: Spatiotemporal trends and source apportionment. Atmos. Chem. Phys. 2014, 14, 12915-12930. [CrossRef]

19. Chen, Y.L.; Hwang, T.L.; Fang, J.Y.; Lan, Y.H.; Chong, K.Y.; Hsieh, P.W. Polysaccharides from Kochia scoparia fruits protect mice from lipopolysaccharide-mediated acute lung injury by inhibiting neutrophil elastase. J. Funct. Foods 2017, 38, 582-590. [CrossRef]

20. Kim, S.J.; Han, D.; Moon, K.D.; Rhee, J.S. Measurement of superoxide dismutase-like activity of natural antioxidants. Biosci. Biotechnol. Biochem. 1995, 59, 822-826. [CrossRef]

21. Murakami, I.; Nakamura, T.; Ishibashi, Y.; Shibuya, R.; Ayano, E.; Morita-Murase, Y.; Nagata, Y.; Kanazawa, H. Simultaneous determination of catechins and procyanidins in bottled tea drinks by LC/MS. Chromatography 2006, 27, 27-33. 
22. Yamashita, Y.; Ashida, H. Functionality of procyanidin. Kagaku to Seibutsu 2016, 54, 747-752. [CrossRef]

23. Hamauzu, Y.; Iijima, E. Polyphenolic composition and antioxidative activity of apple flesh extracts. J. JPN Soc. Food Sci. 1999, 46, 645-651. [CrossRef]

24. Madhan, B.; Krishnamoorthy, G.; Rao, J.R.; Nair, B.U. Role of green tea polyphenols in the inhibition of collagenolytic activity by collagenase. Int. J. Biol. Macromol. 2007, 41, 16-22. [CrossRef] [PubMed]

25. Kawakami, A.; Kayahara, H.; Inbe, T.; Tadasa, K. Inhibition of angiotensin I converting enzyme and collagenase by water extracts of wild and cultivated herbs. J. Fac. Agric. Shinshu Univ. 1994, 31, 97-107.

(C) 2019 by the authors. Licensee MDPI, Basel, Switzerland. This article is an open access article distributed under the terms and conditions of the Creative Commons Attribution (CC BY) license (http://creativecommons.org/licenses/by/4.0/). 